\title{
Time Dilation and Acceleration in Depression
}

\author{
Lachlan Kent ${ }^{1}$, George Van Doorn ${ }^{1}, \&$ Britt Klein ${ }^{2}$ \\ ${ }^{1}$ School of Health Sciences and Psychology, Federation University Australia \\ ${ }^{2}$ Biopsychosocial \& eHealth Research \& Innovation Hub \& DVC-R Portfolio, Federation \\ University Australia
}

\section{Corresponding Author}

Lachlan Kent

School of Health Sciences and Psychology

Federation University Australia

PO Box 663, Ballarat, VIC Australia 3350

Email: lachkent@yahoo.com.au

Phone: +61 405602123 


\begin{abstract}
Background: A recent meta-analysis left open a significant question regarding altered time perception in depression: Why do depressed people overproduce short durations and underproduce longer durations if their present experience is that time flows slowly? Experience and judgement of time do not seem to accord with one another.

Analysis: By excluding two of the six studies on methodological grounds from a previous meta-analysis of medium-length interval productions, and re-analysing the remaining four studies, the present paper finds that subjective time accelerates from initial dilation within present experience (approximately $1 \mathrm{~s}$ duration) to subsequent acceleration within working memory (approximately $30 \mathrm{~s}$ duration) when depressed.

Proposals: It is proposed that depressive time dilation and acceleration refer to the default mode and central executive networks, respectively. The acceleration effect is suggested to occur due to mood congruency between long intervals, boredom, and depression. This mood congruency leads to the automatic recall of intrusive, negative, and non-specific autobiographical long-term memories used to judge intervals from previous experience. Acceleration in working memory then occurs according to the contextual change model of duration estimation.
\end{abstract}

Limitations: The meta-analysis is limited to four studies only, but provides a potential link between time experience and judgement within the same explanatory model.

Conclusions: Similarities between psychological time dilation/acceleration and physical time dilation/acceleration are discussed.

Keywords: Depression, time perception, time experience, time dilation, default mode network, general relativity 
Time Dilation and Acceleration in Depression

\section{Introduction}

Perception and, in particular, time perception are known to be affected by depression (Bech, 1975; Dilling \& Rabin, 1967; Hawkins, French, Crawford, \& Enzle, 1988; Mezey \& Cohen, 1961). While much research has centered on whether depressed individuals' ability to judge time is impaired (Bech, 1975; Bschor et al., 2004; Dilling \& Rabin, 1967; Gallagher, 2012; Kornbrot, Msetfi, \& Grimwood, 2013), strong evidence has been found that time is experienced as passing more slowly when depressed (Kitamura \& Kumar, 1982; Stanghellini et al., 2017; Thönes \& Oberfeld, 2015). This effect, known as depressive time dilation, is well-established and refers to a slowing of temporal flow in conscious experience (Thönes \& Oberfeld, 2015).

The current paper attempts to reconcile two aspects of time perception, namely cognitive judgement and conscious experience, to explain a central problem: In contrast to time experience, depressive time dilation does not seem to impair time judgement straightforwardly. The lack of clarity in the literature concerns methodological differences between experimental protocols and the inability to generalize across all conditions. The following theoretical review, including a re-analysis of a meta-analysis conducted by Thönes and Oberfeld (2015), highlights a potential model of distorted performance when depressed on one specific time judgement task - interval production.

It is important to first outline differences between experimental designs, the cognitive processes implicated in time perception, and how these relate to judgements of time by people experiencing depression. The analysis will then attempt to integrate experience and 
judgement under a single framework that distinguishes between dilation of shorter intervals and acceleration of longer intervals when depressed.

However, before making such strong claims, there are several methodological and conceptual confounds to qualify first (e.g., what is meant by dilation, acceleration, relatively short and relatively long).

\section{Definitions of Time, Dilation, and Acceleration}

The term 'time dilation' is borrowed from physics. It is central to Einstein's (1920) special and general theories of relativity and describes how 'clock' time passes more slowly under certain conditions. For example, physical time is dilated by gravity so that clocks closer to massive gravitational objects operate more slowly than clocks further away (Misner, Thorne, \& Wheeler, 1973). This is called gravitational time dilation.

Psychological time dilation does not measure an interval of clock time in seconds, minutes and so on, but rather how fast or slow an individual experiences the passage of time. The Thönes and Oberfeld (2015) meta-analysis of time perception in depression asserted that time is typically measured using a visual analogue scale from 'very fast' to 'very slow' where, unlike time judgement tasks, "the concept of interval duration does not apply to this type of task" (p. 364). No mechanism to account for the depressive slowing is agreed upon in the literature, and it is not clear whether the mood-disordered time dilation experienced during depression is the same as that induced experimentally (van Wassenhove, Wittmann, Craig, \& Paulus, 2011).

According to the longstanding 'internal clock' model of time perception (Allman, Teki, Griffiths, \& Meck, 2014; Burle \& Casini, 2001; Treisman, 1963; Zakay \& Block, 1995), dilation and acceleration can be interpreted in terms proposed by Glicksohn (2001). 
According to this interpretation, two factors contribute to the length of perceived duration: 1) the size of perceived time units; and 2) the number of time units perceived. Perceived duration is then assumed to be a simple multiple of unit size and number. The perception of larger or fewer time units causes less time to be perceived and so equates to dilation (i.e., time is experienced as passing more slowly). In contrast, the perception of smaller or more time units causes more time to be perceived and so equates to acceleration. The change from dilation to acceleration in depression implies that time units either flow more quickly or become smaller as intervals get longer.

Internal clock interpretations are, however, difficult to simultaneously reconcile with time judgement and experience because measurement of the latter, as above, is not defined with reference to clock-based interval durations (Thönes \& Oberfeld, 2015). We aim to redress this situation by locating time experience within an interval, called the experienced moment, that defines the probable duration of subjective conscious experience. The experienced moment is closely related to conscious sensory-perceptual integration and is generally considered to last approximately 1 - 3 s (Kent, 2019; Pöppel, 1989, 1997; Wang, Lin, Zhou, Pöppel, \& Bao, 2015; White, 2017; Wittmann, 2011). Consciousness is defined here as the subjectivity experienced by conscious beings, which is in contrast to Chalmers' (1996) non-conscious, hypothetical zombies. According to a model proposed by Kent (2019; see Figure 1), the experienced moment is distinct from Wittmann's (2011) shorter timescale functional moments and longer timescale mental presence primarily because: (a) it is a mode of duration perception as opposed to either the sensory-perceptual synchronization of functional moments or extended duration of mental presence; (b) of its reliance on the default mode network as opposed to the salience or central executive networks; and (c) of its critical range of activity being between $200-1250 \mathrm{~ms}$, as opposed to shorter or longer timescales of perceptual $(<200 \mathrm{~ms})$ and working memory $(<30-100 \mathrm{~s})$ processes (Kent, 2019). 


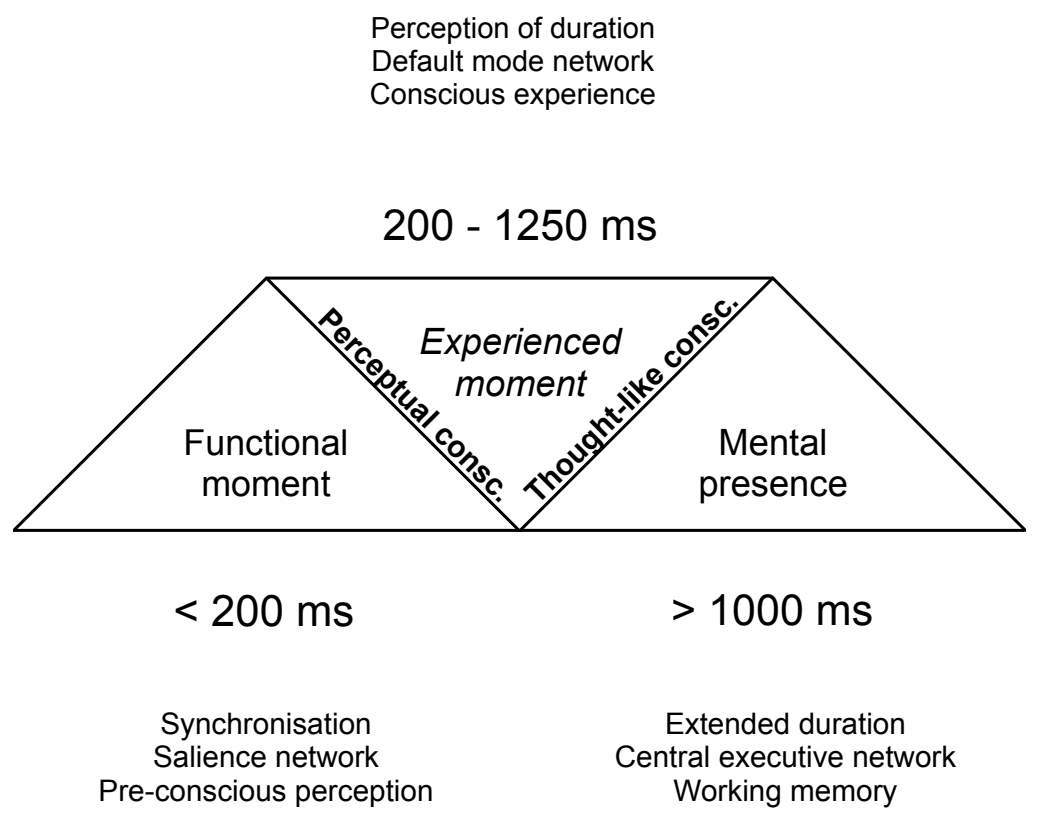

Figure 1. A model proposed by Kent (2019) of the experienced moment as a top-down process spanning approximately $1000 \mathrm{~ms}$ and supported by bottom-up perceptual (functional moment) and working memory (mental presence) processes.

Functional moments are discrete units of time in the milliseconds range that are perceived of as simultaneous and define temporal resolution within a particular sensory modality (Craig, 2009). Audition, for example, has the fastest temporal resolution of $2-3$ ms, whereas vision and tactility are much slower (Wittmann, 2011). Functional moments are therefore aggregated into the experienced moment which, given its relation to consciousness, integrates synchronous sensations into a continuous stream that we experience as consciousness (Kent, 2019).

Mental presence, according to Wittmann (2011), is an extended present centered on working memory (WM) activity that serves as a "temporal platform of multiple seconds 
within which an individual is aware of herself and the environment, where sensory-motor perception, cognition, and emotion are interconnected features of representation leading to phenomenal experience” (p. 6). Previous research places a probable upper limit on mental presence of 30 - $100 \mathrm{~s}$ (Montemayor \& Wittmann, 2014; Wittmann, 2011).

The distinction between the (approximately) $1 \mathrm{~s}$ experienced moment and (approximately) $30 \mathrm{~s}$ mental present is key for current purposes. These are the relatively short and long durations alluded to above and so the assertion is that, when depressed, the experienced moment is dilated whereas mental presence is accelerated. The implication is that time accelerates as intervals increase, an effect which in itself may not be unique to depression. It may be a general feature of time perception that is simply more pronounced for depressed individuals. If it is assumed that the size of Glicksohn's (2001) time units can vary within the same interval, then units at the end of the interval will be relatively small compared to intervals at the beginning of the interval. This temporal compression effect is explained by encoding efficiency models and neural suppression of repeated stimuli (Eagleman \& Pariyadath, 2009).

This is analogous to the situation in ageing whereby a year in later life is only a small proportion of all years lived compared to earlier years, which were a larger proportion at that point in time. Wittmann and Lehnhoff (2005) showed that decades in particular seem to pass more quickly as people age, meaning that decades go from being relatively large to relatively small time units in Glicksohn's (2001) model. If the average human lifespan is considered an interval, in this sense, then the trend from dilation to acceleration may be a more universal facet of time perception that is evident in depression over intervals ranging between the experienced moment and mental presence. In order to demonstrate this effect, empirical studies investigating time judgement in depression must be systematically investigated. 


\section{Assessing Time Judgement}

Common time judgement experiments distinguish between judgements made looking prospectively forwards in time or reconstructed retrospectively looking backwards in time (Zakay, 2014). Experiments are termed prospective or retrospective depending on whether the individual knew that they were timing the interval beforehand (i.e., prospectively) or not (i.e., retrospectively). There are also several judgement methods: (a) estimation of intervals in $\mathrm{ms} / \mathrm{s} / \mathrm{min}$ and so on; (b) reproduction of intervals presented previously; (c) discrimination of the relative length of two or more intervals; (d) bisection of an interval into equal subintervals; and (e) production of an interval from a verbal cue in $\mathrm{ms} / \mathrm{s} / \mathrm{min}$ (Bschor et al., 2004).

Unlike the preceding four conditions, production tasks can only ever be prospective because the individual must be given prior verbal instruction to time the interval. Production methods are also different because they can rely solely on the subjective perception of duration and interoceptive cues (e.g., heartrate or breathing rate), while other tasks must present an interval using some form of exteroceptive cue (e.g., auditory, visual, or tactile) (Bschor et al., 2004). As such, production tasks can be completely devoid of task-related content. The duration of empty and filled intervals are perceived differently, especially for prospective tasks that involve cognitive load on attentional or WM resources (Block, Hancock, \& Zakay, 2010). As such, and unlike other methods, performance on production tasks can be used as a measure of cognitive load (Baldauf, Burgard, \& Wittmann, 2009; Zakay \& Shub, 1998).

The experimental setup is therefore critically important to the assessment of time judgement. Further to this, the duration of intervals used is also critical to whether an effect is detected or not, especially with regards to the distinction between the $1 \mathrm{~s}$ experienced 
moment and $30 \mathrm{~s}$ mental presence (Montemayor \& Wittmann, 2014; Wittmann, 2011). Considering the meta-analytic findings of Thönes and Oberfeld (2015), the authors conflated functional moments, the experienced moment, and mental presence by dividing intervals into ultra-short $(<1 \mathrm{~s})$, short $(1-10 \mathrm{~s})$, medium $(10 \mathrm{~s}-10 \mathrm{~min})$ and long $(>10 \mathrm{~min})$ durations. The studies considered also compared tasks with differing levels of cognitive load. For example, Kornbrot et al. (2013) dissuaded participants from counting by requiring them to recall a 3-digit number, whereas Bschor et al. (2004) allowed participants to count freely.

Our view is that methodological consistency in time perception experiments with depressed participants is crucial to ensure that the findings of several studies can be reasonably compared. As such, a more thorough examination of the relationship between cognition and time perception is required to contextualize methodology.

\section{Cognitive Processes related to Time Perception}

Two factors are known to increase the amount of time judged to have elapsed during a prospective time judgement task: (1) more time is perceived when more attention is allocated to time (Zakay \& Block, 1995; Zakay \& Block, 1996); and (2) more time is perceived when arousal levels are higher (Droit-Volet, Fayolle, \& Gil, 2011; Gil \& Droit-Volet, 2012; Mella, Conty, \& Pouthas, 2011; Yarrow, Haggard, \& Rothwell, 2004). Glicksohn (2001) proposes that attentional effects refer to the size of perceived time units, arousal effects refer to the number of time units, and the two effects multiply together to yield a holistic perception of duration.

While this account of prospective time perception is relatively clear and straightforward, and while it is also known that retrospective intervals are judged as longer when more information is encoded into memory (Block \& Reed, 1978; Rueda \& Schmitter- 
Edgecombe, 2009; Ulbrich, Churan, Fink, \& Wittmann, 2007), it is not well understood how prospective and retrospective models relate to each other. A seminal meta-analysis conducted by Block and Zakay (1997) distinguished between prospective attentional and retrospective memory processing of time, but this conclusion requires further scrutiny for present purposes given that studies using only $5 \mathrm{~s}$ intervals or above were included. The results, therefore, cannot fully account for or distinguish between the $1 \mathrm{~s}$ experienced moment and $30 \mathrm{~s}$ mental presence. While the analysis did distinguish between attentional effects in prospective tasks and short-term memory (STM) effects in relatively short retrospective tasks, it did not consider how WM or long-term memory (LTM) influences prospective intervals in the appropriate range of durations for WM to become relevant. The study therefore did not compare and contrast WM and attentional processes, which have been shown to be related but distinct (Awh, Vogel, \& Oh, 2006; Fougnie, 2008), nor did it investigate how LTM interacts with WM to produce empty intervals in the prospective paradigm.

In the present paper we take the view that longer prospective tasks, and longer production tasks in particular, share some features with retrospective tasks because WM and LTM processes become more influential as the interval increases. The dominant model of interval timing, the information processing model combined with scalar expectancy theory (Allman, Teki, Griffiths, \& Meck, 2014; Buhusi \& Meck, 2005; Gibbon, 1977; Rakitin et al., 1998), proposes that reference memories stored in LTM are used to compare the interval presented with previous experience. For retrospective tasks, because there was no prior knowledge that an interval was to be timed, and therefore no counting or other timing strategy employed, judgement is made by comparing information stored in STM with prior experience stored in LTM. For prospective tasks, which Block and Zakay (1997) aligned with attentional processes, WM must still reconstruct prospective events and temporal experience 
over sufficiently lengthy intervals from memory (Allman et al., 2014; Baldauf et al., 2009; Block \& Zakay, 2006; Zakay \& Shub, 1998).

Fraisse (1984) proposed the simple distinction that durations below $3 \mathrm{~s}$ are directly perceived, whereas longer intervals can only be estimated. The present argument states that prospective judgements of (approximately) $1 \mathrm{~s}$ intervals are perceived within the experienced moment, whereas longer intervals are estimated from WM reconstruction within mental presence (Wittmann, 2011).

Attention and memory are known to interact or overlap in other aspects of temporal cognition. Block and Zakay (2006) explored a similar relationship between interval duration and prospective remembering, which is the ability to remember that a time or event will take place in the future. They proposed that relatively short time-based prospective memory (i.e., remembering to do something at a certain time) uses primarily attentional resources, whereas prospective memory over longer intervals becomes more event-based (i.e., remembering to pay attention to an event) and uses primarily recursive memory resources. Their analysis assumed that prospective remembering "relies on the functioning of well-known attention and memory systems" (p. 25), but that the mode of prospective remembering may change as intervals increase from being time-based to event-based. They called for more research to be done regarding the overlap between prospective remembering and interval production tasks:

\footnotetext{
Research on prospectively made productions has mostly used intervals on the order of seconds, whereas research on prospective remembering has also used intervals on the order of minutes, hours, days, and weeks. Different processes may apply if the target time is hours, days, or weeks in the future. Perhaps the nominally time-based situation becomes more like an event-based situation as the target time is extended further into the future. At the very least, researchers should systematically explore the effects of interval length. (p. 35)
} 
In parallel with these recommendations, we argue that the mode of prospective time judgement in production tasks changes as intervals increase from around $1 \mathrm{~s}$ of the experienced moment into the $30 \mathrm{~s}$ range of mental presence. Increased reliance on memory implies that longer prospective tasks become quasi-retrospective, so a simple binary of prospective-retrospective is inadequate to describe intervals that blend the two. The present paper adds a third mode of judgement called introspective time judgement that is prospective by virtue of the fact that attention is knowingly paid to time, but also quasi-retrospective in that it relies heavily on WM reconstruction.

Under this interpretation, it seems necessary that there is a transitional range somewhere between a few seconds and a few minutes where prospective interval timing tasks switch from being primarily time-based and attentional to primarily event-based and dependent on memory. An introspective transitional range would be consistent with Wittmann's (2011) notion that the experienced moment and mental presence refer to different ranges of activity and also different underlying cognitive processes. Production tasks within this transitional range are potentially relevant to time perception in depression. Sad mood and depression have been shown to interfere with prospective memory performance (Kliegel et al., 2005; Rude, Hertel, Jarrold, Covich, \& Hedlund, 1999), and so there is potential to discern the transition in time perception studies of depressive states.

\section{Time Judgement in Depression}

As above, the recent meta-analysis by Thönes and Oberfeld (2015) found that clinically depressed people tend to overproduce relatively short intervals $(<10 \mathrm{~s})$ and under-produce relatively long intervals ( $>10$ mins). In line with the model illustrated in Figure 1 above, it is proposed here that dilation refers to both the slow experience and delayed judgement of 
intervals within range of the $1 \mathrm{~s}$ experienced moment. Depressed individuals experience a slowing of the $1 \mathrm{~s}$ experienced moment and this effect causes an overproduction of short intervals between $1-10 \mathrm{~s}$ as found by Thönes and Oberfeld (2015). The meta-analysis also defined a medium duration range of $10 \mathrm{~s}-10 \mathrm{~min}$ but, despite the fact that four of the six studies in this range found evidence of underproduction, meta-analytic results did not show an acceleration effect.

However, methodological issues in the two studies that did not show an acceleration effect of medium durations caused the meta-analytic effect-size to be underestimated. Firstly, Tysk (1984) conflated unipolar depressed and bipolar patients into a single "melancholic" group. This is despite current evidence that up to $40 \%$ of bipolar patients experienced mixed episodes of mania with depressive symptoms or, more problematically, depressive episodes with manic symptoms (Fagiolini et al., 2015). Heterogeneous groups were identified as a confound by Bschor et al. (2004) and viewed as a source of contradictory results. A heterogenous unipolar-bipolar group in Tysk (1984) may have moderated any acceleration effect.

Secondly, the Kornbrot, Msetfi, and Grimwood (2013) study only partly satisfied the first meta-analytic criterion employed by Thönes and Oberfeld (2015) to include "data from a group of depressive patients" (p. 362) because it also included an unspecified number of nonclinically depressed individuals with a Beck Depression Inventory (BDI) score as low as 7 (i.e., minimal depression) after median split. As a result, participants in the Kornbrot et al. (2013) study were on average only mildly depressed (BDI, $M=12.5$ ) compared to the moderate-to-severely depressed participants in the Mundt, Richter, van Hees, and Stumpf (1998; BDI, $M=26.8)$, Oberfeld, Thönes, Palayoor, and Hecht (2014; BDI, $M=21.5)$, and Bschor et al. (2004, Hamilton Depression Rating Scale, $M=17.3$ ) studies. It is arguable, 
therefore, that the sample is neither a homogenous group of clinically depressed individuals in itself, nor part of a homogenous set of clinical samples for meta-analysis.

Perhaps due to inhomogenous samples, and as shown in Table 1, the Tysk (1988) and Kornbrot et al. (2013) studies demonstrated effects opposite to the remaining four studies that showed depressive acceleration of productions in the $30 \mathrm{~s}$ WM range of mental presence. A replicated meta-analysis of the remaining four studies, using a classical random effects model and DerSimonian-Laird estimation technique in $\mathrm{R}$, showed that the studies were not significantly heterogenous $\left(Q(3)=3.25, p=.35, \operatorname{Tau}=.084, i^{2}=7.75 \%\right)$ and that the medium-sized acceleration effect was significant (Hedge's $g=.55,95 \% C I=.25-.84, p=$ .0003). Although only four comparable experiments have been performed using a homogenous group of moderate- to severely-depressed participants, their combined effect suggests that, in addition to clinically depressed people experiencing time as dilated in the experienced moment, when those moments are expanded to encompass larger intervals of WM, time becomes accelerated in medium-range $(10-120 \mathrm{~s})$ interval production tasks.

Table 1.

Characteristics of studies included in the Thönes and Oberfeld (2015) meta-analysis, and the strength and direction of under-production (positive statistic) and overproduction (negative statistic) effects of medium length intervals (10 s to 10 mins), where under-production represents an acceleration effect.

\begin{tabular}{lccc}
\hline \multicolumn{1}{c}{ Study } & Homogenous & Intervals & Effect Size \\
& sample & (s) & \\
\hline Bschor et al. (2004) & Yes & $35-90$ & .84 \\
Mundt, Richter, van Hees, and Stumpf (1998) & Yes & $30-120$ & .74
\end{tabular}




$\begin{array}{lccc}\text { Münzel, Gendner, Steinberg, and Raith (1988) } & \text { Yes } & 10 & .37 \\ \text { Oberfeld, Thönes, Palayoor, and Hecht (2014) } & \text { Yes } & 60 & .21 \\ \text { Tysk (1984) } & \text { No } & \mathbf{2 0 - 3 0} & \mathbf{- . 1 9} \\ \text { Kornbrot et al. (2013) } & \text { No } & \mathbf{1 1 - 6 4} & \mathbf{- . 6 7}\end{array}$

\section{Time acceleration in depression}

Assuming an acceleration effect is thus demonstrated meta-analytically, the model proposed below contrasts dilation of present experience and acceleration of immediately recalled mental presence in WM. As illustrated in Figure 1, above, it is possible to dissociate the neural networks associated with dilated experience and accelerated mental presence. Subjective time dilation has been experimentally linked to default mode network activity using intervals in range of the $1 \mathrm{~s}$ experienced moment (van Wassenhove et al., 2011), and depression has also been linked to increased default mode activity via self-referential or emotional processing (Grimm et al., 2009; Sheline et al., 2009). The default mode network, as the name suggests, is a network of brain areas where activity decreases from baseline during the performance of goal-directed behaviours (Raichle et al., 2001). It is also linked to diverse cognitive functions such as autobiographical memory, prospection and theory of mind (Spreng \& Grady, 2010; Spreng, Mar, \& Kim, 2009), temporal awareness (Lloyd, 2012) and consciousness (Vanhaudenhuyse et al., 2009). These associations explain the relationship between the default mode network, conscious experience and time dilation of relatively short intervals as proposed by Kent (2019).

Depressive time acceleration of relatively long intervals, on the other hand, is more likely a function of the central executive network and WM processes, which have also been shown to be impaired by clinical depression (Christopher \& MacDonald, 2005; Joormann \& 
Gotlib, 2008; Rose \& Ebmeier, 2006). As illustrated in Figure 1, above, the default mode and central executive networks affect different aspects of time perception and, during depression, these effects manifest as a slowing of short timescales and quickening of longer timescales. This is an inversion of a psychophysical principle typically applied to reproduction tasks known as Vierordt's law, which states that people tend to over-reproduce shorter durations, under-reproduce longer durations, and be accurate at a duration known as the indifference interval somewhere between 1.5 and 3.5 seconds (Lejeune \& Wearden, 2009). Rather than a relatively narrow and short range of indifference intervals, the proposal here is that there is an indifference range of intervals from a few seconds up to approximately $30 \mathrm{~s}$ representing the transition from time-based to event-based prospective memory (Block \& Zakay, 2006) in the operational range of WM and mental presence (Wittmann, 2011). This means that depressed people may be more accurate at producing particular intervals in the transitional range, while tending to overproduce shorter intervals and under-produce longer intervals.

There is some evidence that this is in fact the case. Although not a clinical sample, Kornbrot et al. (2013) found a "depressive realism effect" in a production task of intervals between 2 and $64 \mathrm{~s}$. People with higher levels of depressive symptoms were more accurate than people with lower symptom levels and were close to accurate at around $30 \mathrm{~s}$, precisely in the range of WM mental presence (Wittmann, 2011). Although this was not a uniformly clinically depressed group, the results are suggestive of increased accuracy over the transitional range. Another study by Bschor et al. (2004) found that clinically depressed people overproduced a $7 \mathrm{~s}$ interval but under-produced a $35 \mathrm{~s}$ interval, suggesting that the indifference interval lies between $20-25 \mathrm{~s}$, again in the transitional range. A further study by Mundt et al. (1998) also found that clinically depressed people significantly under-produced $30 \mathrm{~s}$ durations but not $10 \mathrm{~s}$ durations compared to controls, again suggesting a transitional range around $20 \mathrm{~s}$. Note that the precise mid-point or extent of the transitional range will also 
be partly determined by range of intervals presented in the task and central tendency effects (Buhusi \& Meck, 2005; Lejeune \& Wearden, 2009).

However, a more recent study by Oberfeld et al. (2014) found no differences between depressed and control participants' productions of $0.5 \mathrm{~s}, 2 \mathrm{~s}$, and $60 \mathrm{~s}$ intervals. They also found no effect of depressive time dilation, contrary to the later meta-analysis by two of the same authors (Thönes \& Oberfeld, 2015). The results of the experimental study by Oberfeld et al. (2014) require closer consideration. It is possible, for example, that the production results may have been confounded by entrainment to the target intervals which were presented up to 15 times in the preceding verbal estimation condition. The essential feature of interval production is that time is judged according to internal cues only, which may have been overridden by reference to the same intervals having been externally cued may times prior to the production task. There was also no graduation of intervals between $2 \mathrm{~s}$ and $60 \mathrm{~s}$, meaning that the $0.5 \mathrm{~s}$ and $2 \mathrm{~s}$ intervals may have influenced each other through a central tendency effect known to cause an averaging between nearby intervals presented in the same set (Buhusi \& Meck, 2005; Lejeune \& Wearden, 2009). The production of the two short intervals in the Oberfeld et al. (2014) study may have migrated towards the mean of the two already-entrained intervals (i.e., $1.25 \mathrm{~s}$ ).

Overall, there is some evidence for (Bschor et al., 2004; Kornbrot et al., 2013; Mundt et al., 1998; Münzel et al, 1988) and less evidence against (Oberfeld et al., 2014; Tysk, 1984) a transition from over- to under-production in the range of WM mental presence when depressed. In the absence of definitive evidence, the only recourse is to direct and indirect evidence presented above: (a) meta-analysis of production-task data requires a transition (Thönes \& Oberfeld, 2015); (b) research into prospective memory implies a transition (Block \& Zakay, 2006); (c) time perception research provides a WM mechanism for transition (Kent, 2019; Wittmann, 2011); and (d) depression research suggests that productions around the 30 
s interval may be critical to understanding time judgement when depressed (Bschor et al., 2004; Kornbrot et al., 2013; Mundt et al., 1998). The key point to make is that time experience and judgement when depressed do not map onto each other for intervals significantly longer than the experienced moment and, given that depressive time dilation can explain overproduction of short intervals, it is reasonable to assume that depressive time acceleration explains the under-production of longer intervals.

\section{Possible Explanations}

We argue that the re-analysed meta-analytic findings of Thönes and Oberfeld (2015) require explanations for three related phenomena: (a) why production tasks are more affected than other tasks when depressed; (b) why perception of empty intervals accelerates over introspective intervals when depressed; and (c) what it means to dilate short intervals and accelerate longer intervals.

\subsection{Why are production tasks primarily affected by depression?}

First, production tasks appeared to be the most affected because production is the only task where the duration is not supplied to the participant. Production tasks are uniquely reliant on generating an interval 'subjectively', as it were, from a verbal cue (Bschor et al., 2004). The model illustrated in Figure 1, above, proposes that interoceptive cues produced by the salience network, such as heartbeat and breathing rate, create synchronous emotional moments that are then integrated into the temporal flow of conscious experience by the default mode network (Craig, 2009; Kent, 2019). Activity in the default mode network has been associated with awareness of time (Lloyd, 2012), subjective time dilation (van 
Wassenhove, Wittmann, Craig, \& Paulus, 2011), and depression (Sheline et al., 2009). The default mode is also generally active when task-related cognitive processing load is low (Buckner, Andrews-Hanna, \& Schacter, 2008; Raichle, 2015) and when thoughts are predominantly related to the self (Sheline et al., 2009). It is therefore implicated in a range of cognitive functions including prospection and autobiographical memory (Spreng \& Grady, 2010; Spreng, Mar, \& Kim, 2009).

Production tasks serve as a measure of cognitive load during a concurrent task (Baldauf et al., 2009; Zakay and Block, 1998), so there is a clear distinction between default mode and central executive activity during production tasks, with the latter being responsible for processing task-relevant cognitive load and the former responsible for generating taskirrelevant temporal awareness (Lloyd, 2012). In comparison to other time perception methods, production tasks are better able to distinguish between effects of major brain networks relevant to conscious time experience and time judgement over intervals of different length (Kent, 2019).

The mechanism for acceleration between default mode and central executive time perception during depression is attributed to the function of the salience network as a switch directing information to either of the other two networks (Goulden et al., 2014; Sridharan, Levitin, \& Menon, 2008). During a cognitively-demanding task, highly-integrated sensory information is directed to the central executive network for processing, but during off-task periods of rest information is directed to the default mode network (Sridharan, et al., 2008). Depression severity has been linked to poor connectivity within and between the three major brain networks including: (a) aberrant connectivity within the anterior insula cortex, a key node of the salience network; (b) aberrant connectivity between the default mode and central executive networks; and (c) increased connectivity between the right anterior insula cortex and the default mode network (Monoliu et al., 2014). The salience network also remains 
more active during off-task rest periods when depressed (Wiebking et al., 2010), meaning interoceptive information is more salient and there is greater awareness of time (Lloyd, 2012). This latter effect leads to depressive time dilation of the experienced moment and the sense that time is passing (present continuous tense) slowly. An additional mechanism is required to explain the opposite acceleration effect that occurs when reconstructing longer intervals in WM to give the sense that time has passed (present perfect tense) quickly when producing introspective intervals with low cognitive load.

\subsection{Why do empty intervals accelerate as they get longer when depressed?}

The second point to explain concerns the gradual acceleration of time productions through the transitional range $(<30 \mathrm{~s})$ of introspective WM and mental presence when depressed. Two cognitive mechanisms are proposed: (a) mood congruency between boredom, depression, and relatively long, empty intervals (Farmer \& Sundberg, 1986; Zakay, 2014); and (b) reduced WM capacity within the episodic buffer as a result of altered autobiographical memory.

First, boredom and depression have been shown to overlap significantly (Farmer \& Sundberg, 1986) and, along with default mode network activity (Buckner et al., 2008; Raichle, 2015), boredom is generally defined as periods when information processing loads are low or sub-optimal (Zakay, 2014). A long, empty production task interval is one such period when information processing load is low, compared to an interval full of distractor tasks, for example. Danckert and Allman (2005) showed that boredom-proneness becomes relevant to time perception for intervals between $15-60 \mathrm{~s}$, with a trend evident in the $30 \mathrm{~s}$ transition range of WM. During these boring production intervals, evidence suggests that depressed people will: (a) experience greater intrusion of irrelevant thoughts that distract 
from the task at hand (Ellis \& Moore, 1999); and (b) recall information that is moodcongruent between storage and recall (Bower, 1981; Ellis \& Moore, 1999). This means that higher levels of depression-relevant information are more likely to be recalled during a boring, empty interval.

Second, this mood congruency effect is likely to affect the type of long-term reference memories depressed people use to produce intervals in the transitional range (Block \& Zakay, 2006). Reference memory is a component of information processing models of interval timing such as attentional-gate and scalar timing models (Allman et al., 2014; Zakay \& Block, 1995). Previous research points to autobiographical long-term memories (ALTMs) as being central to the depressive experience: (a) depressed people are better able to recall negative than positive events (Lloyd \& Lishman, 1975); (b) stressful memories tend to be more intrusive when depressed, and recovery from depression is linked to the degree of intrusion by, and avoidance of, stressful memories (Brewin, Reynolds, \& Tata, 1999); (c) memories tend to be nonspecific or over-general when depressed (Williams \& Scott, 1988; Williams, Teasdale, Segal, \& Soulsby, 2000); and (d) the over-general nature of depressive autobiographical memory is closely related to WM capacity and executive control (Dalgleish et al., 2007). This means that during relatively long, empty interval productions likely to make boredom-proneness salient (Danckert \& Allman, 2005), it is expected that depressed individuals are more likely to experience intrusive, negative, and non-specific ALTMs that affect their WM capacity.

ALTMs affect WM processes and mental presence through the limited-capacity 'episodic buffer' introduced by Baddeley (2000) as an additional component of the multimodal theory of WM that also includes the visuospatial sketchpad, phonological loop, and central executive. Further to this, Montemayor and Wittmann (2014) detailed how ALTMs are incorporated into the episodic buffer and the $30 \mathrm{~s}$ WM mental present: 
The distinction between episodic short-term memory and long-term memory of all kinds (e.g., semantic, episodic, autobiographical) is crucial for the characterization of this third type of present...While short-term memory would specify the availability of contents for cognitive integration, the contents of autobiographical long-term memory form the impression of a stable self that endures through time as part of the experience of reminiscing the past and planning for the future. That is, the contents of autobiographical memory are instantiated within the boundary of the mental present defined as the temporal window of working memory. (p. 333, italics added).

If autobiographical memory is "instantiated within" (p. 333) the "temporal window of working memory" (p. 333) of approximately 30 s duration, then introspective judgements of intervals within the range of $30 \mathrm{~s}$ must incorporate ALTM within WM processes. This reinforces the notion that the binary distinction between attention and memory processes (Block and Zakay,1997; Zakay, 2014) does not hold for introspective time judgement.

Removing the distinction for introspective intervals does not, however, change the nature of attention and memory effects on time judgement. The contextual change model (Block \& Reed, 1978) of memory-based judgements still holds, which states that more time is judged to have elapsed when more changes were encoded during, or recalled after, an interval. For introspective production tasks, judgement is partly based on the amount of contextual change expected to occur during the interval based on reference memories previously encoded for similar durations.

As such, depressed individuals are likely to refer to non-specific ALTMs that contain fewer contextual changes than non-depressed individuals. When employing WM to produce a relatively long, empty and potentially boring interval (Danckert \& Allman, 2005), the memories they refer to must themselves be relatively 'empty' if they are to overestimate how much time has passed and stop the interval early. This effect is the mechanism proposed to 
account for depressive time acceleration of introspective intervals in the approximate $30 \mathrm{~s}$ range of WM.

Overall, this proposal suggests that the limited-capacity episodic WM buffer of a depressed person is more likely to be filled by intrusive, negative, nonspecific ALTMs than somebody who is not depressed. For economy, the term negative memory mass will be used to refer to these intrusive, negative, nonspecific ALTMs from now on. The term 'mass' conveys the nonspecific nature of memories that lack detail even though they 'fill' the episodic buffer. As such, negative memory mass will both: (a) fill WM capacity and the episodic buffer; and (b) act as the reference memory used to judge the length of the interval as per attentional-gate and scalar timing models of interval judgement (Allman et al., 2014; Zakay \& Block, 1995). However, due to its nonspecific nature, memory mass does not represent a large amount of contextual change and so, according to the contextual change model for remembered durations (Block \& Zakay, 1997; Block \& Reed, 1978), the amount of change expected to occur during introspective intervals will be less and the interval will be stopped early in the approximate $30 \mathrm{~s}$ WM range of mental presence (Wittmann, 2011).

\subsection{What does it mean to dilate shorter intervals and accelerate longer intervals?}

We, therefore, propose that depressed individuals' judgement of time accelerates from a state of relative time dilation within the $1 \mathrm{~s}$ experienced present to a state of relative time acceleration within the $30 \mathrm{~s}$ mental present during empty, boring intervals. To summarize this process step-by-step: (a) when people are depressed (or bored), time appears to flow slowly within the $1 \mathrm{~s}$ experienced present because more attention is paid to time (Block \& Reed, 1978; Thönes \& Oberfeld, 2015; Zakay, 2014; Zakay \& Block, 1995; Zakay \& Block, 1996) and interoceptive information is more salient (Manoliu et al., 2014; Wiebking et al., 2010); 
(b) during longer empty intervals, depressed people automatically recall more negative memory mass as reference memories within their WM episodic buffer (Dalgleish et al., 2007; Lloyd \& Lishman, 1975; Williams et al., 2000); and (c) due to the higher proportion of less changeable memories available in the WM episodic buffer, the contextual change model predicts that intervals around $30 \mathrm{~s}$ will not be expected to last as long and therefore become accelerated (Baddeley, 2000; Block \& Zakay, 2006; Block \& Reed, 1978; Montemayor \& Wittmann, 2014; Wittmann, 2011; Zakay, 2014).

With this in mind, the relation between time judgement and time experience is made understandable. Prospective temporal flow is experienced within a short $1 \mathrm{~s}$ window and is the flow referred to when people report time as: (a) slow when depressed or bored (Thönes \& Oberfeld, 2015; Zakay, 2014); or (b) fast when experiencing a flow state during an intrinsically rewarding and challenging experience (Csikszentmihalyi, 1975; Nakamura \& Csikszentmihalyi, 2002, 2014). Introspective temporal flow is that which is mentally estimated within the longer $30 \mathrm{~s}$ window and what people refer to when they feel they are under 'time pressure', an effect that has been demonstrated to accelerate interval productions between 30 - 90 s (Rattat, Matha, \& Cegarra, 2018), precisely in the range of WM mental presence. Even though time pressure itself can promote positive or negative effects on wellbeing (Widmer, Semmer, Kälin, Jacobshagen, \& Meier, 2012), it has nevertheless been shown to be associated with negative mood, emotional irritation, and depression (Höge, 2009; Roxburgh, 2004 \& 2012; Teuchmann, Totterdell, \& Parker, 1999).

Depression may therefore affect both prospective and introspective temporal flow by slowing down experience while applying time pressure (acceleration) to mental presence. Time pressure can be defined as a situation likely to increase arousal and so increase the number of time units perceived during an interval (Glicksohn, 2001). Alternatively, acceleration in depression could also be defined as an impairment of sustained attention, 
which has been shown to affect depressed people's production of relatively long intervals (Sévigny, Everett, \& Grondin, 2003), and so to gradually decrease the size of corresponding time units (Glicksohn, 2001). Within this interpretation, it is important to clearly identify whether arousal or attention is the factor accelerating or dilating time perception. Glicksohn (2001) formulates the distinction in terms of the limits of time perception and the experience of 'timelessness':

In fact, there are two theoretical ways to achieve the state of timelessness. As suggested above, one path is via meditation (hypoarousal), inducing a longer and longer subjective time unit, which becomes the limiting case of the flow of time as all attentional resources are allocated in inward reflection...But consider the case of hyperarousal (e.g., extreme stress, such as experienced in near death situations)...As the train of subjective time units accelerates, with each subjective time unit becoming smaller and smaller in extension, then the limit is reached, much as when a temporal "critical flicker fusion" is realized...The individual subjective time units then become fused into one. (p. 12)

Assuming that depressive time dilation is a form of timelessness (i.e., time has slowed almost to a stop) then there are two potential causes of overproduction of short intervals: 1) 'large' time units; or 2) 'slow' pacemaker. There are also two possible causes of underproduction of long intervals: 1) 'small' time units; or 2) 'fast' pacemaker.

Sevigny et al. (2003) showed that the acceleration of longer intervals when depressed is due to poor sustained attention, implying that attention may be the primary driver of depressive time acceleration and depression. Consistent with this view, van Wassenhove et al. (2011) concluded that dilation of short $1 \mathrm{~s}$ intervals is not attributable to arousal of the salience network but rather 'self-referential processes' of the default mode network. Selfreferential processes are very similar to Glicksohn’s (2001) “inward reflection” and so, on both these accounts, subjective time dilation/acceleration in depression is most likely 
attentional. This means that, in Glicksohn's (2001) model, time units become smaller as time experience accelerates in depression. The current paper proposes that this gradual 'shrinking' of time units occurs because reference LTMs are 'shorter' when reconstructed in WM according to the low prevalence of contextual change due to memory mass.

In this sense, time is directly experienced as flowing slowly but estimated as though it is flowing relatively quickly, implying that judgement type (production), modality (experience versus judgement), and duration (short versus long) are all necessary to show the effect in depression. The rate of subjective temporal flow is a function of interval, in other words. To coin a term, time gradually flies when you are depressed. To some extent, this acceleration principle unifies dynamics of time judgement and time experience under a single time 'sense' (Bschor et al., 2004). Judgement and experience arise from the same overall perception that blends attentional, WM and LTM processes.

Depression is interpreted as the tendency to experience this acceleration effect to a greater degree so that there is a greater discrepancy between short-range prospective and medium-range introspective temporal flow. This discrepancy entails greater variability in experience and judgement due to acceleration of the internal timing mechanisms, whether classical (Allman et al., 2014) or Bayesian (Shi, Church, \& Meck, 2013), as intervals increase. Recent theories support such a notion. For example, Northoff (2018) recently proposed that the spatiotemporal characteristics of depression may be influenced by increased neuronal variability in the default mode network. Acceleration over time is therefore the key concept linking the phenomenal and cognitive aspects of time perception when depressed, as opposed to the instantaneous dilation of time in the experienced moment.

This situation is alluded to in the following excerpt from Block and Zakay's (1997) meta-analysis of prospective and retrospective timing tasks: 


\begin{abstract}
Most theorists think that retrospective duration judgments increase as a function of the amount of stored and retrieved information, or storage size..., the number of remembered changes..., the number of encoded and available contextual changes..., or the degree of segmentation of events during the time period... Thus, theorists typically propose memory-based models. Attention to time may play little or no role in remembered duration unless a person has little information to process, frequent feelings of boredom, and so on. Such conditions may arouse a temporal motive. (p. 185)
\end{abstract}

The congruency between boring, empty production tasks and depression is precisely one of those situations likely to "arouse a temporal motive" (p. 185) in which attention to time within the $1 \mathrm{~s}$ experienced present becomes important to the memory processes required to estimate longer durations. Slow prospective temporal flow (i.e., depressive time dilation) prompts more memory mass to fill the WM episodic buffer, increasing the relative number of perceived perceptual changes compared to the number of contextual changes present in reference memory. It is this relativistic trade-off between perception and memory that is of key interest for future research.

\title{
8. Acceleration and Relativity
}

With these three explanations in mind, the broader implications of introspective acceleration can be discussed, in particular the relationship between physical and psychological definitions of time. Before proceeding with this analysis, it is important to emphasise that time perception research need not 'reinvent the wheel' when it comes to modelling and explaining temporal phenomena. Physics has been grappling with the multifarious nature of time but, perhaps unlike psychological theory, it has arrived at a consensual model of time and space, namely relativity theory. Psychological time shares this central feature with physical time in that it appears to be relative (i.e., speed up and slow down) to the context. 
Some work has already been done to align psychological and physical theories of time. For example, Buhusi and Meck (2009) showed that the relative length of target and distractor variables affects judgement. They found that rats' timing behaviours varied depending on the length of the target interval $(10-90 \mathrm{~s})$ versus the relative salience of a distracting interval gap (1, 3 and $10 \mathrm{~s})$. The longer the gap was in relation to the target, the more likely the rats were to 'reset' the psychological 'clock' used to time the target interval, as opposed to merely 'stopping' or continuing to 'run' it. Resetting the psychological clock caused greater delay in timing judgements than either stopping or running. The gap length was judged relative to the length of the entrained criterion. The authors explained this phenomenon with reference to Einstein's (1920) 'special' theory of relativity by concluding that multiple clocks arrive at different judgements of interval length depending on their own temporal properties (i.e., trained interval length).

Einstein's (1920) special theory of relativity describes time dilation effects under conditions of constant motion (i.e., no acceleration), such that a moving clock ticks slowly from the point of view of an observer 'at rest'. The amount of dilation is directly proportionate to the motion of a moving clock relative to a stationary clock. Buhusi and Meck (2009) drew an analogy between the delayed activity of psychological 'clocks' and the dilation of physical clocks in uniform motion. They also proposed the rate of decay of WM activity as a causal mechanism for relativistic judgement. The larger an interval gap is relative to the target interval, the more quickly WM activity should decay during the interval gap. The more quickly WM decays, the more likely the 'clock' will be reset and the more judgement will be delayed. WM decay is therefore an analogue of motion with relativistic effects.

The above depiction of time dilation and acceleration in depression shows a similar interaction between interval and timing except that, in this case, there is no distractor interval 
and the delay/dilation can reverse to become anticipation/acceleration. Comparison with special relativity is not able to explain this latter phenomenon because, given that both the moving and stationary clocks register each other as dilated, special relativity is symmetrical with regards to dilation (Einstein, 1920). This is analogous to time dilation during depression when both the depressed individual and objective clock judge the other as delayed, dilated or slow. However, time acceleration in depression means that the relationship between the depressed observer and objective clock time is no longer symmetrical. The depressed individual still judges experienced time as dilated but the clock judges the depressed individual as accelerated. From the clock's point of view, the depressed person changes from responding too slowly (i.e., overproduction) to responding too quickly (i.e., underproduction).

This asymmetrical feature of depressive time acceleration is more reminiscent of Einstein's (1920) 'general' theory of relativity which explains how gravitational time dilation varies (i.e., accelerates) according to two variables: mass and distance. An observer at a fixed interval from a central mass will measure a clock that is closer to the mass as dilated and a clock that is further away as accelerated. The difference in dilation/acceleration between closer and further clocks is also greater when there is more central mass. At the extreme, a clock close to the surface of a supermassive object (i.e., a black hole) will appear to slow to the point of stopping from the point of view of an observer further away (Misner, Thorne, \& Wheeler, 1973). As such, general relativity describes a gravitational field as a field of acceleration determined by mass and distance.

The analogy here is that: (a) greater levels of boredom and/or depression create greater levels of non-specific memory mass; and (b) time dilation at short interval ranges of the $1 \mathrm{~s}$ experienced moment accelerates over longer intervals of $30 \mathrm{~s}$ mental presence. From the clock's point of view, the timing of a dilated/depressed individual accelerates as the 
interval increases, which is precisely the situation in a gravitational field when clocks accelerate with distance from a central mass.

Therefore, the principle of depressive time dilation/acceleration outlined above accords with Einstein's (1920) general relativity theory of gravity because it explains properties of psychological time in terms of the same three variables: mass, interval (distance), and acceleration. The greater the tendency towards depression and/or boredom during introspective production intervals, the greater the memory mass recalled as a reference memory used to judge the interval in WM, and the more quickly a clock will measure the response compared to shorter prospective intervals.

\section{Limitations}

The meta-analysis of four studies requires further data to confirm the existence of depressive time acceleration. This limitation notwithstanding, it must be emphasized that the analogy with physics is not a literal or even figurative association between depression, darkness, down-ness, and gravity. The explanation is made purely in technical and conceptual terms of dilation, acceleration, mass, interval, and reference frames. Recent phenomenological work has been done describing the varieties of depressive temporal experience and they constitute a somewhat separate argument to the technical analogy being proposed here (Ratcliffe, 2012; Stanghellini et al., 2017). Application of a strict analogy is similar to theoretical approaches using quantum probability theory to model judgement errors (Busemeyer, Pothos, Franco, \& Trueblood, 2011), similarity judgements (Pothos, Busemeyer, \& Trueblood, 2013), and causal reasoning (Trueblood \& Busemeyer, 2012). All that these theories borrow from physics is the conceptual and statistical framework to show that psychological and physical processes follow qualitatively and quantifiably similar patterns. The current explanation of 
time dilation and acceleration in depression uses the same approach to model time experience and judgement.

Previous research besides Buhusi and Meck's (2009) has explored the association between perception and relativity theory. Müsseler (1999) reviewed evidence for a relativistic interpretation of visual perceptual contraction phenomena, the corollary of time dilation, and concluded that more evidence was required to fit information-processing accounts of motion perception within the conceptual framework of special relativity. Some early progress has been made. For example, time dilation and length contraction effects have been demonstrated for objects moving across the visual field (Caelli, Hoffman, \& Lindman, 1978), but studies such as these are not conclusively in favor of a strict analogy between physical and psychological relativity.

\section{Conclusion}

Meta-analysis of depressed samples' performance on time production tasks suggests that, in contrast to the initial dilation of conscious time experience over short intervals, time judgement of longer intervals accelerates in WM. Whereas dilated experience is most likely due to activity in the default mode network, the acceleration effect can be explained in terms of mood congruency, automatic recall of non-specific reference memories, and the contextual change model of duration estimation. This explanation expands previous work on the relativistic nature of time judgement in WM by showing how psychological time accelerates in a similar way to physical time. It also unites time experience and judgement under a single theoretical model of time perception.

\section{Acknowledgements}


The authors thank Liz Temple who assisted with the early preparation and structure of the paper, Rose Ferguson who helped with the meta-analysis, and Terri Caelli and Mark Bennet who helped draft previous versions. We also thank and acknowledge the Australian Government Research Training Program for supporting the first author. 


\section{References}

Allman, M. J., Teki, S., Griffiths, T. D., \& Meck, W. H. (2014). Properties of the Internal Clock: First- and Second-Order Principles of Subjective Time. Annual Review of Psychology, 65(1), 743-771. doi:10.1146/annurev-psych-010213-115117

Awh, E., Vogel, E., \& Oh, S.-H. (2006). Interactions between attention and working memory. Neuroscience, 139(1), 201-208. doi:10.1016/j.neuroscience.2005.08.023

Baddeley, A. (2000). The episodic buffer: a new component of working memory? Trends in Cognitive Sciences, 4(11), 417-423. doi:10.1016/S1364-6613(00)01538-2

Baddeley, A. (2012). Working memory: theories, models, and controversies. Annual Review of Psychology, 63, 1-29. doi:10.1146/annurev-psych-120710-100422

Baldauf, D., Burgard, E., \& Wittmann, M. (2009). Time perception as a workload measure in simulated car driving. Applied ergonomics, 40(5), 929-935. doi:10.1016/j.apergo.2009.01.004

Bech, P. (1975). Depression: influence on time estimation and time experiments. Acta Psychiatrica Scandinavica, 51(1), 42-50. doi.org/10.1016/j.apergo.2009.01.004

Block, R., \& Zakay, D. (1997). Prospective and retrospective duration judgments: A metaanalytic review. Psychonomic Bulletin \& Review, 4(2), 184-197. doi:10.3758/bf03209393

Block, R., \& Zakay, D. (2006). Prospective remembering involves time estimation and memory processes. In Timing the future: The case for a time-based prospective memory (pp. 25-49): World Scientific.

Block, R. A., Hancock, P. A., \& Zakay, D. (2010). How cognitive load affects duration judgments: A meta-analytic review. Acta Psychologica, 134(3), 330-343. 
Block, R. A., \& Reed, M. A. (1978). Remembered duration: Evidence for a contextualchange hypothesis. Journal of Experimental Psychology: Human Learning and Memory, 4(6), 656-665.

Bower, G. H. (1981). Mood and memory. American psychologist, 36(2), 129-148.

Brewin, C. R., Reynolds, M., \& Tata, P. (1999). Autobiographical memory processes and the course of depression. Journal of Abnormal Psychology, 108(3), 511-517.

Bschor, T., Ising, M., Bauer, M., Lewitzka, U., Skerstupeit, M., Müller-Oerlinghausen, B., \& Baethge, C. (2004). Time experience and time judgment in major depression, mania and healthy subjects. A controlled study of 93 subjects. Acta Psychiatrica Scandinavica, 109(3), 222-229.

Buhusi, C. V., \& Meck, W. H. (2005). What makes us tick? Functional and neural mechanisms of interval timing. Nature Reviews, 6(10), 755-765.

Buckner, R. L., Andrews-Hanna, J. R., \& Schacter, D. L. (2008). The brain's default network. Annals of the New York Academy of Sciences, 1124(1), 1-38. doi:10.1196/annals. 1440.011

Buhusi, C. V., \& Meck, W. H. (2009). Relativity Theory and Time Perception: Single or Multiple Clocks? PLoS ONE, 4(7), e6268. doi:10.1371/journal.pone.0006268

Burle, B., \& Casini, L. (2001). Dissociation between activation and attention effects in time estimation: Implications for internal clock models. Journal of Experimental Psychology: Human Perception and Performance, 27(1), 195-205. doi:10.1037/00961523.27.1.195

Busemeyer, J. R., Pothos, E. M., Franco, R., \& Trueblood, J. S. (2011). A quantum theoretical explanation for probability judgment errors. Psychological Review, 118(2), 193-218. doi:10.1037/a0022542 
Caelli, T., Hoffman, W., \& Lindman, H. (1978). Subjective Lorentz transformations and the perception of motion. Journal of the Optical Society of America, 68(3), 402-411. doi: $10.1364 /$ josa. 68.000402

Chalmers, D. J. (1996). The conscious mind: In search of a fundamental theory: Oxford University Press.

Christopher, G., \& MacDonald, J. (2005). The impact of clinical depression on working memory. Cognitive neuropsychiatry, 10(5), 379-399.

Craig, A. D. (2009). Emotional moments across time: a possible neural basis for time perception in the anterior insula. Philosophical Transactions of the Royal Society B: Biological Sciences, 364(1525), 1933-1942.

Csikszentmihalyi, M. (1975). Play and intrinsic rewards. Journal of humanistic psychology. 15(3), 41-63. http://dx.doi.org/10.1177/002216787501500306

Dalgleish, T., Williams, J. M. G., Golden, A.-M. J., Perkins, N., Barrett, L. F., Barnard, P. J., ... Tchanturia, K. (2007). Reduced specificity of autobiographical memory and depression: the role of executive control. Journal of Experimental Psychology: General, 136(1), 23-42.

Danckert, J. A., \& Allman, A.-A. A. (2005). Time flies when you're having fun: Temporal estimation and the experience of boredom. Brain and Cognition, 59(3), 236-245.

Dilling, C. A., \& Rabin, A. I. (1967). Temporal experience in depressive states and schizophrenia. Journal of Consulting Psychology, 31(6), 604-608. doi:10.1037/h0025160

Droit-Volet, S., Fayolle, S. L., \& Gil, S. (2011). Emotion and time perception: Effects of film-induced mood. Frontiers in Integrative Neuroscience, 5(33), 1-9. doi:10.3389/fnint.2011.00033 
Eagleman, D., \& Pariyadath, V. (2009). Is subjective duration a signature of coding efficiency? Philosophical Transactions of the Royal Society B: Biological Sciences, 364(1525), 1841-1851.

Einstein, A. (1920). Relativity: The special and general theory. New York: Henry Holt.

Ellis, H. C., \& Moore, B. A. (1999). Mood and memory. In T. Dalgleish \& M. Power (Eds.), Handbook of cognition and emotion (pp. 193-210): John Wiley \& Sons.

Fagiolini, A., Coluccia, A., Maina, G., Forgione, R. N., Goracci, A., Cuomo, A., \& Young, A. H. (2015). Diagnosis, epidemiology and management of mixed states in bipolar disorder. CNS drugs, 29(9), 725-740.

Farmer, R., \& Sundberg, N. D. (1986). Boredom proneness - the development and correlates of a new scale. Journal of personality assessment, 50(1), 4-17.

Fougnie, D. (2008). The relationship between attention and working memory. In N. B. Johansen (Ed.), New research on short-term memory, (pp. 1-45.). Nova Science Publishers.

Fraisse, P. (1984). Perception and estimation of time. Annual Review of Psychology, 35(1), 137.

Gallagher, S. (2012). Time, emotion, and depression. Emotion Review, 4(2), 127-132.

Gibbon, J. (1977). Scalar expectancy theory and Weber's law in animal timing. Psychological Review, 84(3), 279-325. doi:10.1037/0033-295x.84.3.279

Gil, S., \& Droit-Volet, S. (2012). Emotional time distortions: The fundamental role of arousal. Cognition \& Emotion, 26(5), 847-862. doi:10.1080/02699931.2011.625401

Glicksohn, J. (2001). Temporal cognition and the phenomenology of time: A multiplicative function for apparent duration. Consciousness and Cognition, 10(1), 1-25.

Goulden, N., Khusnulina, A., Davis, N. J., Bracewell, R. M., Bokde, A. L., McNulty, J. P., \& Mullins, P. G. (2014). The salience network is responsible for switching between the 
default mode network and the central executive network: replication from DCM. NeuroImage, 99, 180-190. doi:10.1016/j.neuroimage.2014.05.052

Grimm, S., Boesiger, P., Beck, J., Schuepbach, D., Bermpohl, F., Walter, M., . . Northoff, G. (2009). Altered negative BOLD responses in the default-mode network during emotion processing in depressed subjects. Neuropsychopharmacology, 34(4), 932. doi:10.1038/npp.2008.81

Hawkins, W. L., French, L. C., Crawford, B. D., \& Enzle, M. E. (1988). Depressed affect and time perception. Journal of Abnormal Psychology, 97(3), 275-280. doi:10.1037/0021$843 x .97 .3 .275$

Höge, T. (2009). When work strain transcends psychological boundaries: An inquiry into the relationship between time pressure, irritation, work-family conflict and psychosomatic complaints. Stress and Health: Journal of the International Society for the Investigation of Stress, 25(1), 41-51.

Joormann, J., \& Gotlib, I. H. (2008). Updating the contents of working memory in depression: Interference from irrelevant negative material. Journal of Abnormal Psychology, 117(1), 182-192.

Kent, L. (2019). Duration Perception Versus Perception Duration: A Proposed Model for the Consciously Experienced Moment. Timing \& Time Perception, 7(1), 1-14.

Kitamura, T., \& Kumar, R. (1982). Time passes slowly for patients with depressive state. Acta Psychiatrica Scandinavica, 65(6), 415-420. doi:10.1111/j.16000447.1982.tb00865.x

Kliegel, M., Jäger, T., Phillips, L., Federspiel, E., Imfeld, A., Keller, M., \& Zimprich, D. (2005). Effects of sad mood on time-based prospective memory. Cognition \& Emotion, 19(8), 1199-1213. 
Kornbrot, D. E., Msetfi, R. M., \& Grimwood, M. J. (2013). Time perception and depressive realism: Judgment type, psychophysical functions and bias. PLoS ONE, 8(8), 1-9. doi:10.1371/journal.pone.0071585

Lejeune, H. (1998). Switching or gating? The attentional challenge in cognitive models of psychological time. Behavioural Processes, 44(2), 127-145.

Lejeune, H., \& Wearden, J. H. (2009). Vierordt's The Experimental Study of the Time Sense (1868) and its legacy. European Journal of Cognitive Psychology, 21(6), 941-960. doi:10.1080/09541440802453006

Lloyd, D. (2012). Neural correlates of temporality: Default mode variability and temporal awareness. Consciousness and Cognition, 21(2), 695-703.

Lloyd, G. G., \& Lishman, W. A. (1975). Effect of depression on the speed of recall of pleasant and unpleasant experiences. Psychological medicine, 5(2), 173-180.

Manoliu, A., Meng, C., Brandl, F., Doll, A., Tahmasian, M., Scherr, M., . . Bäuml, J. (2014). Insular dysfunction within the salience network is associated with severity of symptoms and aberrant inter-network connectivity in major depressive disorder. Frontiers in human neuroscience, 7, 930.

Mella, N., Conty, L., \& Pouthas, V. (2011). The role of physiological arousal in time perception: Psychophysiological evidence from an emotion regulation paradigm. Brain and Cognition, 75(2), 182-187. doi:10.1016/j.bandc.2010.11.012

Mezey, A. G., \& Cohen, S. I. (1961). The effect of depressive illness on time judgment and time experience. Journal of Neurology, Neurosurgery \& Psychiatry, 24, 269-270. doi:10.1136/jnnp.24.3.269

Misner, C. W., Thorne, K. S., \& Wheeler, J. A. (1973). Gravitation: W. H. Freeman \& Company. 
Montemayor, C., \& Wittmann, M. (2014). The varieties of presence: hierarchical levels of temporal integration. Timing \& Time Perception, 2(3), 325-338.

Mundt, C., Richter, P., van Hees, H., \& Stumpf, T. (1998). Zeiterleben und Zeitschätzung depressiver Patienten. Der Nervenarzt, 69(1), 38-45. doi:10.1007/s001150050236

Münzel, K., Gendner, G., Steinberg, R., \& Raith, L. (1988). Time estimation of depressive patients: The influence of interval content. European Archives of Psychiatry \& Neurological Sciences, 237(3), 171-178. doi:10.1007/bf00451286

Müsseler, J. (1999). Perceiving and measuring of spatiotemporal events. In J. S. Jordan (Ed.), Modeling consciousness across the disciplines. (pp. 95-112). Lanham, MD US: University Press of America.

Nakamura, J., \& Csikszentmihalyi, M. (2002). The concept of flow. In C. R. Snyder \& S. J. Lopez (Eds.), Handbook of positive psychology. (pp. 89-105). New York, NY US: Oxford University Press.

Nakamura, J., \& Csikszentmihalyi, M. (2014). The concept of flow. In Flow and the foundations of positive psychology (pp. 239-263): Springer.

Northoff, G. (2018). Why Do We Need Psychopathology? From the Brain's Resting State to “Spatiotemporal Psychopathology" of Depression. In Understanding Depression (pp. 145-152): Springer.

Oberfeld, D., Thönes, S., Palayoor, B. J., \& Hecht, H. (2014). Depression does not affect time perception and time-to-contact estimation. Frontiers in Psychology, 5, 810.

Pöppel, E. (1989). A hierarchical model of human time perception. International Journal of Psychophysiology, 7(2-4), 357-359. doi:http://dx.doi.org/10.1016/0167$\underline{8760(89) 90292-4}$

Pöppel, E. (1997). A hierarchical model of temporal perception. Trends in Cognitive Sciences, 1(2), 56-61. 
Pothos, E. M., Busemeyer, J. R., \& Trueblood, J. S. (2013). A quantum geometric model of similarity. Psychological Review, 120(3), 679-696. doi:10.1037/a0033142

Raichle, M. E., MacLeod, A. M., Snyder, A. Z., Powers, W. J., Gusnard, D. A., \& Shulman, G. L. (2001). A default mode of brain function. Proceedings of the National Academy of Sciences, 98(2), 676-682.

Raichle, M. E. (2015). The brain's default mode network. Annual Review of Neuroscience, $38,433-447$.

Rakitin, B. C., Gibbon, J., Penney, T. B., Malapani, C., Hinton, S. C., \& Meck, W. H. (1998). Scalar expectancy theory and peak-interval timing in humans. Journal of Experimental Psychology: Animal Behavior Processes, 24(1), 15-33.

Ratcliffe, M. (2012). Varieties of temporal experience in depression. Journal of Medicine \& Philosophy, 37(2), 114-138.

Rattat, A.-C., Matha, P., \& Cegarra, J. (2018). Time flies faster under time pressure. Acta Psychologica, 185, 81-86.

Rose, E., \& Ebmeier, K. (2006). Pattern of impaired working memory during major depression. Journal of Affective Disorders, 90(2), 149-161.

Roxburgh, S. (2004). “There Just Aren't Enough Hours in the Day': The Mental Health Consequences of Time Pressure. Journal of health and social behavior, 45(2), 115131.

Roxburgh, S. (2012). Parental time pressures and depression among married dual-earner parents. Journal of Family Issues, 33(8), 1027-1053.

Rude, S. S., Hertel, P. T., Jarrold, W., Covich, J., \& Hedlund, S. (1999). Depression-related impairments in prospective memory. Cognition \& Emotion, 13(3), 267-276. 
Rueda, A. D., \& Schmitter-Edgecombe, M. (2009). Time estimation abilities in mild cognitive impairment and Alzheimer's disease. Neuropsychology, 23(2), 178-188. doi:10.1037/a0014289

Sévigny, M.-C., Everett, J., \& Grondin, S. (2003). Depression, attention, and time estimation. Brain and Cognition, 53(2), 351-353. doi:http://dx.doi.org/10.1016/S02782626(03)00141-6

Sheline, Y. I., Barch, D. M., Price, J. L., Rundle, M. M., Vaishnavi, S. N., Snyder, A. Z., . . . Raichle, M. E. (2009). The default mode network and self-referential processes in depression. Proceedings of the National Academy of Sciences, 106(6), 1942-1947.

Shi, Z., Church, R. M., \& Meck, W. H. (2013). Bayesian optimization of time perception. Trends in Cognitive Sciences, 17(11), 556-564. doi:http://dx.doi.org/10.1016/j.tics.2013.09.009

Spreng, R. N., \& Grady, C. L. (2010). Patterns of brain activity supporting autobiographical memory, prospection, and theory of mind, and their relationship to the default mode network. Journal of cognitive neuroscience, 22(6), 1112-1123.

Spreng, R. N., Mar, R. A., \& Kim, A. S. (2009). The common neural basis of autobiographical memory, prospection, navigation, theory of mind, and the default mode: a quantitative meta-analysis. Journal of cognitive neuroscience, 21(3), 489510.

Sridharan, D., Levitin, D. J., \& Menon, V. (2008). A critical role for the right fronto-insular cortex in switching between central-executive and default-mode networks. Proceedings of the National Academy of Sciences, 105(34), 12569-12574.

Stanghellini, G., Ballerini, M., Presenza, S., Mancini, M., Northoff, G., \& Cutting, J. (2017). Abnormal time experiences in major depression: an empirical qualitative study. Psychopathology, 50(2), 125-140. 
Teuchmann, K., Totterdell, P., \& Parker, S. K. (1999). Rushed, unhappy, and drained: an experience sampling study of relations between time pressure, perceived control, mood, and emotional exhaustion in a group of accountants. Journal of occupational health psychology, 4(1), 37.

Thönes, S., \& Oberfeld, D. (2015). Time perception in depression: A meta-analysis. Journal of Affective Disorders, 175, 359-372.

Treisman, M. (1963). Temporal discrimination and the indifference interval: Implications for a model of the 'internal clock'. Psychological Monographs: General and Applied, 77(13), 1-31. doi:10.1037/h0093864

Trueblood, J., \& Busemeyer, J. R. (2012). A quantum probability model of causal reasoning. Frontiers in Psychology, 14(3), 1-13. doi:10.3389/fpsyg.2012.00138

Tysk, L. (1984). Time perception and affective disorders. Perceptual and Motor Skills, 58(2), 455-464. doi:10.2466/pms.1984.58.2.455

Ulbrich, P., Churan, J., Fink, M., \& Wittmann, M. (2007). Temporal reproduction: Further evidence for two processes. Acta Psychologica, 125(1), 51-65. doi:http://dx.doi.org/10.1016/j.actpsy.2006.06.004

van Wassenhove, V., Wittmann, M., Craig, A., \& Paulus, M. (2011). Psychological and Neural Mechanisms of Subjective Time Dilation. Frontiers in Neuroscience, 5(56), 110. doi:10.3389/fnins. 2011.00056

Vanhaudenhuyse, A., Noirhomme, Q., Tshibanda, L. J.-F., Bruno, M.-A., Boveroux, P., Schnakers, C., . . . Brichant, J.-F. (2009). Default network connectivity reflects the level of consciousness in non-communicative brain-damaged patients. Brain, 133(1), 161-171. 
Wang, L., Lin, X., Zhou, B., Pöppel, E., \& Bao, Y. (2015). Subjective present: a window of temporal integration indexed by mismatch negativity. Cognitive Processing, 16(1), 131-135.

White, P. A. (2017). The three-second "subjective present": A critical review and a new proposal. Psychological Bulletin, 143(7), 735.

Widmer, P. S., Semmer, N. K., Kälin, W., Jacobshagen, N., \& Meier, L. L. (2012). The ambivalence of challenge stressors: Time pressure associated with both negative and positive well-being. Journal of Vocational Behavior, 80(2), 422-433.

Wiebking, C., Bauer, A., De Greck, M., Duncan, N. W., Tempelmann, C., \& Northoff, G. (2010). Abnormal body perception and neural activity in the insula in depression: an fMRI study of the depressed "material me". The World Journal of Biological Psychiatry, 11(3), 538-549.

Williams, J., \& Scott, J. (1988). Autobiographical memory in depression. Psychological medicine, 18(3), 689-695.

Williams, J., Teasdale, J. D., Segal, Z. V., \& Soulsby, J. (2000). Mindfulness-based cognitive therapy reduces overgeneral autobiographical memory in formerly depressed patients. Journal of Abnormal Psychology, 109(1), 150-155.

Wittmann, M. (2011). Moments in Time. Frontiers in Integrative Neuroscience, 5(66), 1-9. doi:10.3389/fnint.2011.00066

Wittmann, M., \& Lehnhoff, S. (2005). Age effects in perception of time. Psychological reports, 97(3), 921-935.

Yarrow, K., Haggard, P., \& Rothwell, J. C. (2004). Action, arousal, and subjective time. Consciousness and Cognition, 13(2), 373-390. doi:http://dx.doi.org/10.1016/j.concog.2003.10.006 
Zakay, D. (2014). Psychological time as information: the case of boredom. Frontiers in Psychology, 5(917). doi:10.3389/fpsyg.2014.00917

Zakay, D., \& Block, R. A. (1995). An attentional-gate model of prospective time estimation. Time and the dynamic control of behavior, 167-178.

Zakay, D., \& Block, R. A. (1996). The role of attention in time estimation processes. In M.

A. Pastor \& J. Artieda (Eds.), Time, internal clocks and movement. (pp. 143-164). Amsterdam Netherlands: North-Holland/Elsevier Science Publishers.

Zakay, D., \& Shub, J. (1998). Concurrent duration production as a workload measure. Ergonomics, 41(8), 1115-1128. 